\title{
THERE IS A DETECTABLE IMMUNE ACTIVITY IN THE ORBITS OF ALL PATIENTS DIAGNOSED WITH GRAVES' DISEASE REGARDLESS OF LATER DEVELOPMENT OF GRAVES' ORBITOPATHY
}

\section{OBJECTIVES}

Graves' orbitopathy (GO) is a common complication of Graves' disease (GD), the development of which can not be predicted at the time of diagnosis of uncomplicated GD. It is crucial to detect orbital immune activity as active $\mathrm{GO}$ tends to respond to immunosuppressive therapy, whereas inactive disease does not.

We assumed that orbital autoimmune activity is predictable using orbital 99mTc-labelled diethylenetriamine pentaacetic acid SPECT (DTPA.) The procedure is relatively inexpensive, reproducible, and represents little burden for the patient (Figure 1.)

We aimed to determine whether any orbital autoimmune activity can be identified in patients who do not develop GO during their follow-up.

\section{MATERIALS AND METHODS}

Fifty-four orbits of 27 patients newly diagnosed with GD were entered into the study. Patients with present GO were excluded. None of the patients had received antithyroid drugs or ophthalmic measures before entering the study. An initial DTPA was performed and DTPA uptake as sign of disease activity calculated in each case. SPECT was repeated during follow-up if clinical signs of GO occurred, and a final SPECT was performed at the end of the follow-up period, after one year. Twenty orbits of control patients who underwent DTPA of the hands for Raynaud's phenomenon served as controls.

\section{RESULTS}

During the one-year follow-up period 6 out of the total of 27 patients $(22 \%)$ were diagnosed with newly developed GO. The mean DTPA uptake at the time of diagnosis of GD of the 6 patients who later developed GO (and before any treatment) was 10.45 \pm 1.72 $\mathrm{MBq} / \mathrm{cm}^{3}$ (mean $\pm S D$ ). This result was statistically not different from the DTPA uptake of the 42 orbits of patients who were not affected by $G O$ during the follow up period $\left(9.18 \pm 1.18 \mathrm{MBq} / \mathrm{cm}^{3}\right)$ but the difference was not significant. However, the DTPA uptakes of both patient groups were higher than that of the control group $\left(7.7 \pm 2.44 \mathrm{MBq} / \mathrm{cm}^{3}\right)$, the difference being significant, $p=0.013$ and $p=0.032$ for those who later developed $G O$ and who did not, respectively.

Acknowledgements

The work is supported by a grant from the TÁMOP-4.2.2.A-11/1/KONV2012-0031 project and the TÁMOP 4.2.4. A/2-11-1-2012-0001 'National Excellence Program.' The TÁMOP project is co-financed by the European Union and the European Social Fund.
Figure 1. DTPA images of Graves' patients without clinically active GO (evaluated with CAS) showed a mild positivity due to an ongoing immunoactivity
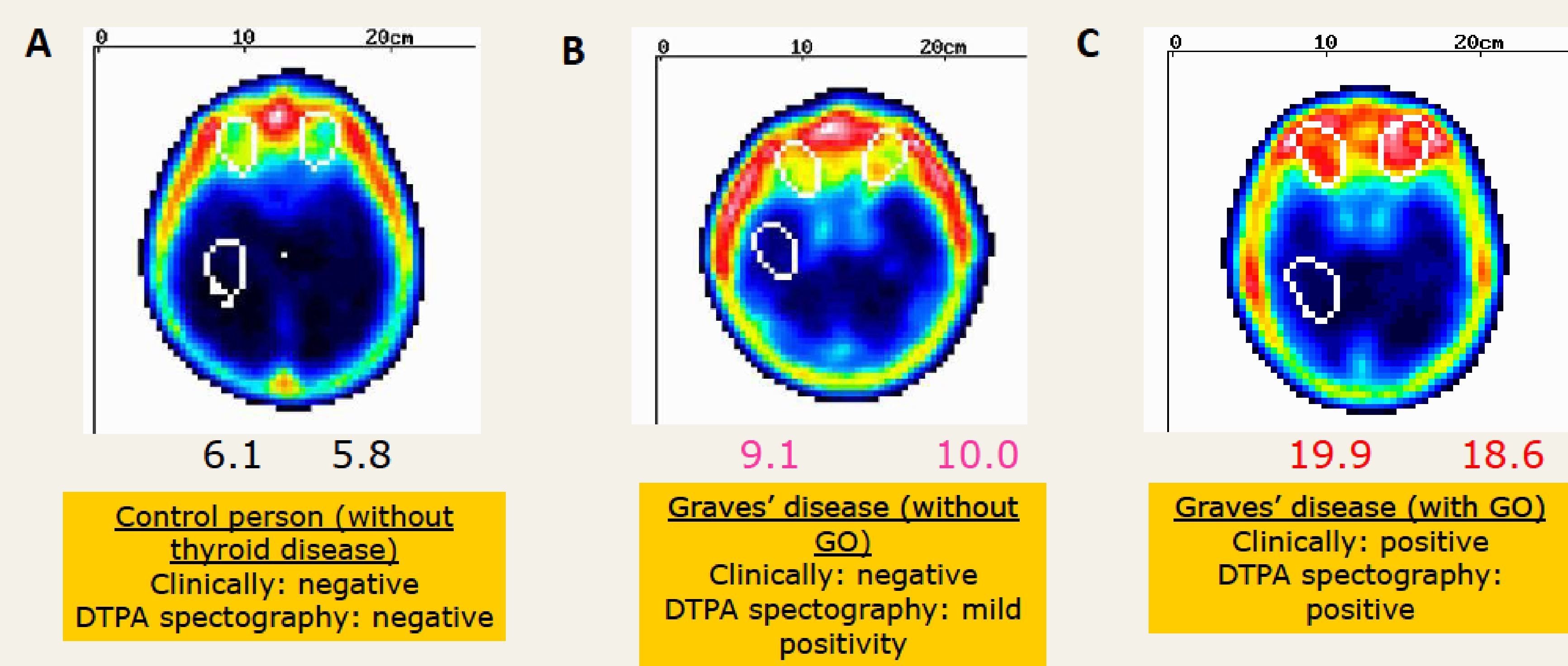

Figure 2. The initial DTPA values of Graves' patients without GO were significantly different from the values of healthy normal subjects and the values of patients who later developed GO

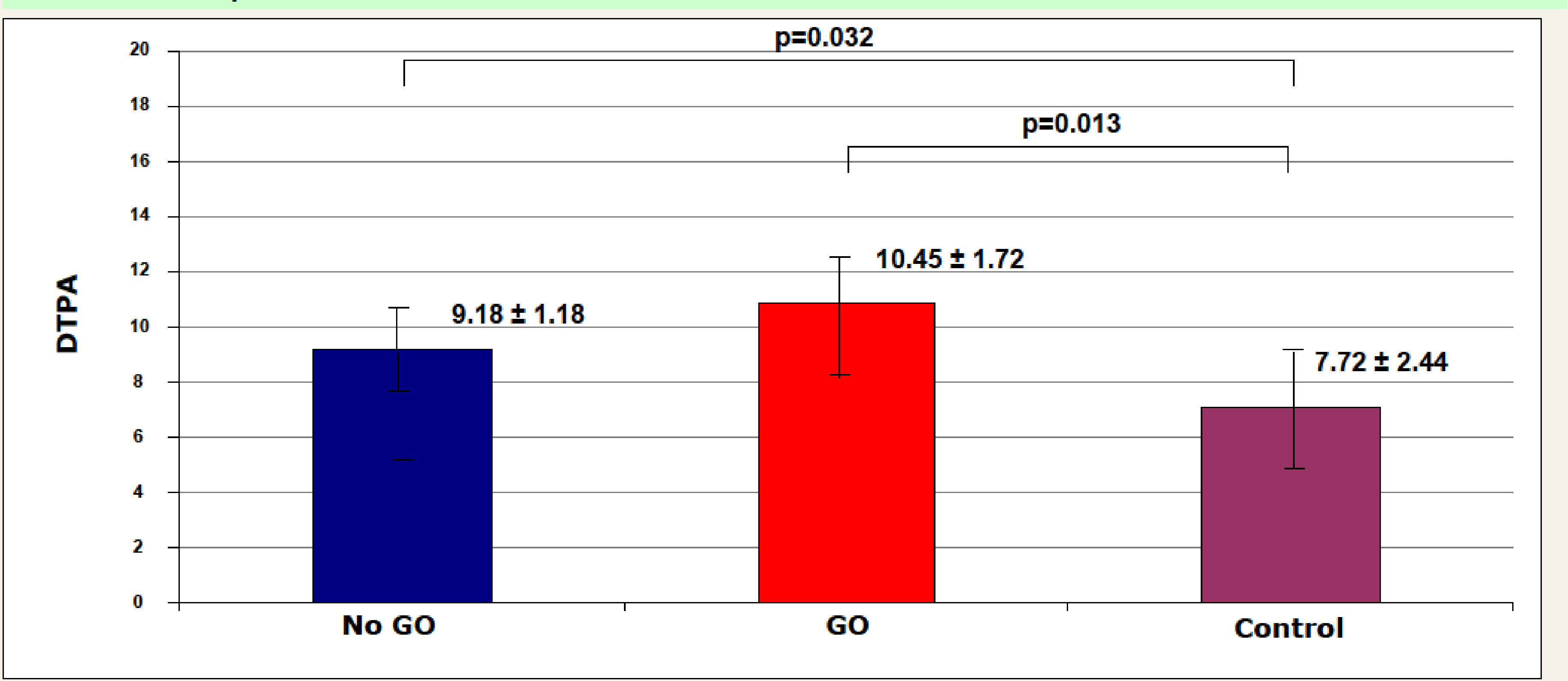

The retrobulbar DTPA activity obtained at the end of the one-year long follow-up period of the healthy normal subjects, those GD patients who did not develop GO and those who developed $\mathrm{GO}$ differed significantly, being $7.7 \pm 2.67 \mathrm{MBq} / \mathrm{cm}^{3}, \quad 9.63 \pm 3.30 \mathrm{MBq} / \mathrm{cm}^{3}$ and 12.1 $\pm 4.41 \mathrm{MBq} / \mathrm{cm}^{3}$, respectively (Figure 2.)

\section{CONCLUSIONS}

$22 \%$ of the patients developed GO during the one year long follow up, DTPA performed at an early stage of GD failed to predict the later development of $\mathrm{GO}$. In patients with GD not complicated by $\mathrm{GO}$ at disease start, mildly elevated DTPA uptake was detected, irrespective of later development of GO. This important finding confirms that there is a substantial autoimmune activity in the orbits all patients with GD, even from a very early stage of disease; however, it will progress to overt GO only in $20-50 \%$ of the patients.

According to our results, the subsequent onset of $\mathrm{GO}$ in GO-naïve GD patients cannot be predicted by DTPA. Why this orbital autoimmunity remains subclinical in the majority of the patients or progresses into clinically detectable $\mathrm{GO}$ in others, remains unclear. This uniform ongoing subclinical inflammation may be a reversible early stage of GO. The complex causes that will lead later in a several percentage of patients to develop GO eye symptoms need to be further investigated and elucidated. 